PROCEEDINGS OF THE

AMERICAN MATHEMATICAL SOCIETY

Volume 126, Number 9, September 1998, Pages 2747-2753

S $0002-9939(98) 04600-0$

\title{
$M$-IDEALS OF COMPACT OPERATORS ARE SEPARABLY DETERMINED
}

\author{
EVE OJA
}

(Communicated by Palle E. T. Jorgensen)

\begin{abstract}
We prove that the space $K(X)$ of compact operators on a Banach space $X$ is an $M$-ideal in the space $L(X)$ of bounded operators if and only if $X$ has the metric compact approximation property (MCAP), and $K(Y)$ is an $M$-ideal in $L(Y)$ for all separable subspaces $Y$ of $X$ having the MCAP. It follows that the Kalton-Werner theorem characterizing $M$-ideals of compact operators on separable Banach spaces is also valid for non-separable spaces: for a Banach space $X, K(X)$ is an $M$-ideal in $L(X)$ if and only if $X$ has the MCAP, contains no subspace isomorphic to $\ell_{1}$, and has property $(M)$. It also follows that $K(Z, X)$ is an $M$-ideal in $L(Z, X)$ for all Banach spaces $Z$ if and only if $X$ has the MCAP, and $K\left(\ell_{1}, X\right)$ is an $M$-ideal in $L\left(\ell_{1}, X\right)$.
\end{abstract}

\section{INTRODUCTION}

A (closed) subspace $Y$ of a Banach space $X$ is called an $M$-ideal if there exists a projection $P$ on the dual space $X^{*}$ such that $\operatorname{Ker} P=Y^{\perp}$, and $\left\|x^{*}\right\|=\left\|P x^{*}\right\|+$ $\left\|x^{*}-P x^{*}\right\|$ for all $x^{*} \in X^{*}$.

Already more than twenty years, many authors have studied conditions for the space $K(X)$ of compact operators on $X$ to be an $M$-ideal in the space $L(X)$ of bounded operators (see [8, pp. 333-336] for a brief history and references) with the main aim to characterize those Banach spaces $X$ for which $K(X)$ is an $M$-ideal in $L(X)$. Some years ago, N. J. Kalton and D. Werner [10] showed that, for a separable Banach space $X, K(X)$ is an $M$-ideal in $L(X)$ if and only if $X$ has the metric compact approximation property (MCAP), contains no subspace isomorphic to $\ell_{1}$, and has property $(M)$ (property $(M)$, which is an internal geometric property of $X$, will be defined in Section 2 below). Since the method of proof of this characterization implies the separability of $X$, the question whether the Kalton-Werner theorem is also valid for non-separable $X$ remained open.

In Section 1 of the present note, we prove that $M$-ideals of compact operators are separably determined: $K(X)$ is an $M$-ideal in $L(X)$ if and only if $X$ has the MCAP, and $K(Y)$ is an $M$-ideal in $L(Y)$ for all separable subspaces $Y$ of $X$ having the MCAP. In Section 2, this enables us to extend the Kalton-Werner theorem to non-separable spaces $X$. This also enables us to show that $K(Z, X)$ is an $M$-ideal in $L(Z, X)$ for all Banach spaces $Z$ if and only if $X$ has the MCAP, and $K\left(\ell_{1}, X\right)$ is an $M$-ideal in $L\left(\ell_{1}, X\right)$ (cf. Section 3 ).

Received by the editors February 14, 1997.

1991 Mathematics Subject Classification. Primary 46B28, 47D15, 46B20.

The author was partially supported by the Estonian Science Foundation Grant 3055. 
Let us fix some more notation. In a Banach space $X$, we denote the closed unit ball by $B_{X}$. For a set $A \subset X$, its norm closure is denoted by $\bar{A}$, its linear span by span $A$, and its convex hull by conv $A$. The set of all weak* strongly exposed points of $B_{X^{*}}$ is denoted by $w^{*}$-sexp $B_{X^{*}}$, and the identity operator of $X$ is denoted by $I_{X}$ or simply by $I$. Recall that $X$ is said to have the MCAP if there is a net in $B_{K(X)}$ converging strongly to $I$. (This means that $K(X)$ contains a left 1-approximate unit (cf. e.g. [8, p. 294]).)

\section{M-IDEALS OF COMPACT OPERATORS}

The following characterization of $M$-ideals of compact operators will be needed below to prove that $M$-ideals of compact operators are separably determined.

Theorem 1. Let $X$ be a Banach space. Then $K(X)$ is an $M$-ideal in $L(X)$ if and only if $X$ an $M$-ideal in $X^{* *}$, and for all $S \in B_{K(X)}$ there is a net $\left(K_{\alpha}\right)$ in $B_{K(X)}$ such that $K_{\alpha} \rightarrow I$ strongly and

$$
\limsup \left\|S+I-K_{\alpha}\right\| \leq 1
$$

Proof. The necessity is well known (cf. [11] or [8, p. 291] together with [19] or [8, p. 299]). Sufficiency. Recall that $K(X)$ is an $M$-ideal in $L(X)$ if and only if $K(X)$ is an $M$-ideal in $\mathcal{L}=\operatorname{span}(K(X) \cup\{I\})$ (cf. [14] or [8, p. 299], or [9] for separable $X)$. Recall also that the MCAP of $X$ implies the existence of a linear norm preserving extension operator $\Phi: K(X)^{*} \rightarrow \mathcal{L}^{*}$ (cf. e.g. [12]). This makes it possible to consider the topology $\sigma=\sigma\left(\mathcal{L}, \Phi\left(K(X)^{*}\right)\right)$. In [18], it is essentially proved (for a simpler proof cf. [6]) that $K(X)$ is an $M$-ideal in $\mathcal{L}$ if and only if for all $S \in B_{K(X)}$ and $T \in B_{\mathcal{L}}$ there is a net $\left(L_{\alpha}\right)$ in $B_{K(X)}$ such that $\left(L_{\alpha}\right)$ is $\sigma$-convergent to $T$ and

$$
\lim \sup \left\|S+T-L_{\alpha}\right\| \leq 1 .
$$

Consider $T=K+\lambda I \in B_{\mathcal{L}}$ (with $K \in K(X)$ ). Note that $|\lambda| \leq 1$ because otherwise $K$ would be invertible. Therefore $\lambda=r e^{i \varphi}$ with $r \in[0,1]$. For $e^{-i \varphi} S$, choose the net $\left(K_{\alpha}\right)$. Since $X$ has the unique extension property (following from the fact that $X$ is an $M$-ideal in $\left.X^{* *}\right)$, we have $x^{*}\left(K_{\alpha}^{*} x^{*}\right) \rightarrow x^{* *}\left(x^{*}\right)$ for all $x^{*} \in X^{*}$, $x^{* *} \in X^{* *}$ (cf. [5] or [8, p. 118]). Set $L_{\alpha}=K_{\alpha} T=K_{\alpha} K+\lambda K_{\alpha}$. Then $L_{\alpha} \in B_{K(X)}$,

$$
x^{* *}\left(L_{\alpha}^{*} x^{*}\right)=\left(K^{* *} x^{* *}\right)\left(K_{\alpha}^{*} x^{*}\right)+\lambda x^{* *}\left(K_{\alpha}^{*} x^{*}\right) \rightarrow x^{* *}\left(T^{*} x^{*}\right),
$$

i.e. $\left(x^{* *} \otimes x^{*}\right)\left(L_{\alpha}-T\right) \rightarrow 0$ for all $x^{* *} \otimes x^{*} \in \mathcal{L}^{*}$, and (since $\left\|K_{\alpha} K-K\right\| \rightarrow 0$ )

$$
\begin{aligned}
\limsup \left\|S+T-L_{\alpha}\right\| & =\limsup \left\|S+r e^{i \varphi} I-r e^{i \varphi} K_{\alpha}\right\| \\
& =\limsup \left\|e^{-i \varphi} S+r I-r K_{\alpha}\right\| \\
& \leq r \lim \sup \left\|e^{-i \varphi} S+I-K_{\alpha}\right\|+1-r \\
& \leq 1 .
\end{aligned}
$$

We know that $B_{X^{*}}=\overline{\operatorname{conv}}\left(w^{*}\right.$-sexp $\left.B_{X^{*}}\right)$ and $X^{*}$ has the Radon-Nikodým property (because $X$ is an $M$-ideal in $X^{* *}$; cf. e.g. [8, pp. 126, 127], the latest implying $K(X)^{*}=\overline{X^{* *} \otimes X^{*}}$ by [4, Theorem 1]. Hence, $L_{\alpha} \rightarrow T$ in the $\sigma$-topology whenever $(\Phi g)\left(L_{\alpha}-T\right) \rightarrow 0$ for all $g=x^{* *} \otimes x^{*} \in K(X)^{*}$ with $x^{* *} \in X^{* *}$ and $x^{*} \in w^{*}-\operatorname{sexp} B_{X^{*}}$. By the proof of [12, Lemma 3.4 (a)], such a $g$ has a unique norm-preserving extension (to the whole $L(X)$ ). Thus, $\Phi g=x^{* *} \otimes x^{*} \in \mathcal{L}^{*}$, and the result follows. 
Remark 1. It is known (cf. [14] or [8, p. 299], or [9] for separable $X$ ) that $K(X)$ is an $M$-ideal in $L(X)$ if and only if there is a net $\left(K_{\alpha}\right)$ in $B_{K(X)}$ such that both $K_{\alpha} \rightarrow I_{X}$ and $K_{\alpha}^{*} \rightarrow I_{X^{*}}$ strongly, and limsup $\left\|S+I-K_{\alpha}\right\| \leq 1$ for all $S \in B_{K(X)}$.

Remark 2. The following is clear from the proof of Theorem 1: if $X$ is an $M$-ideal in $X^{* *}$ (in particular, if $K(X)$ is an $M$-ideal in $L(X)$ ), and $K_{\alpha} \rightarrow I$ strongly for some net $K_{\alpha} \in B_{K(X)}$, then $K_{\alpha} \rightarrow I$ in the $\sigma\left(L(X), \Phi\left(K(X)^{*}\right)\right)$-topology for any linear norm preserving extension operator $\Phi: K(X)^{*} \rightarrow L(X)^{*}$.

The next theorem is the main result of the present note.

Theorem 2. Let $X$ be a Banach space. Then $K(X)$ is an $M$-ideal in $L(X)$ if and only if $X$ has the $M C A P$, and $K(Y)$ is an $M$-ideal in $L(Y)$ for all separable subspaces $Y$ of $X$ having the $M C A P$.

Proof. The necessity is well known (cf. [14] or [8, p. 301], or [9] for separable $X$ ). Sufficiency. We shall apply Theorem 1 . To prove that $X$ is an $M$-ideal in $X^{* *}$, we need to show that every separable subspace of $X$ is an $M$-ideal in its bidual (cf. [13] or [8, p. 115]). Consider a separable subspace $Y$ of $X$. Since $X$ has the MCAP, $Y$ is contained in a separable subspace $Z$ of $X$ having the MCAP (the proof of this fact is the same as of the similar fact for the metric approximation property (cf. e.g. [17, p. 606])). Thus, $K(Z)$ is an $M$-ideal in $L(Z)$, which implies that $Z$ is an $M$-ideal in $Z^{* *}$. But then also its subspace $Y$ is an $M$-ideal in $Y^{* *}$.

Let us now make the following observation. If $K(Y)$ is an $M$-ideal in $L(Y)$ for a separable Banach space $Y$, and $K_{n} \rightarrow I_{Y}$ strongly for some sequence $\left(K_{n}\right) \subset B_{K(Y)}$, then, for all $S \in B_{K(Y)}$ and $\varepsilon>0$, there is some $K \in \operatorname{conv}\left\{K_{1}, K_{2}, \ldots\right\}$ such that $\left\|S-K+I_{Y}\right\| \leq 1+\varepsilon / 2$. [Due to Remark 2, the proof of this fact is the same as of the similar assertion about Banach spaces being $M$-ideals in their biduals in [13, Proposition 2.8, (i) $\Longrightarrow$ (ii)] (cf. also [8], p. 113), only using instead of the weak* topology the $\sigma\left(L(Y), \Phi\left(K(Y)^{*}\right)\right.$ )-topology (where $\Phi: K(Y)^{*} \rightarrow L(Y)^{*}$ is the (unique linear) norm preserving extension operator).]

We denote by $s_{o p}$ the strong operator topology on $L(X)$, and suppose that the condition of Theorem 1 is not satisfied: for some $S \in B_{K(X)}$, there is no such net. Then there are $\varepsilon>0$ and a convex $s_{o p}$ neighbourhood $U_{0}$ of $I$ such that

$$
\|S-K+I\|>1+\varepsilon \quad \forall K \in B_{K(X)} \cap U_{0} .
$$

For all $n \in \mathbb{N}$, denote by $\Lambda_{n}$ a finite $\varepsilon / 4$-net in the subset $\left\{\left(\lambda_{1}, \ldots, \lambda_{n}\right): \lambda_{k} \geq\right.$ $\left.0, \lambda_{1}+\cdots+\lambda_{n}=1\right\}$ of $\ell_{1}^{n}$. Let $\left(K_{\alpha}\right)_{\alpha \in \mathcal{A}}$ be a net in $B_{K(X)}$ converging to $I$ in the $s_{o p}$. We shall follow some ideas from the proofs of Proposition 2.8, (iii) $\Longrightarrow$ (iv), in [13] (cf. [8, p. 114]) and Theorem 18.2 in [17, p. 606] to pick a sequence $\alpha_{1}, \alpha_{2}, \ldots$ in $\mathcal{A}$ and to define a separable subspace $Y \subset X$ so that $S(X) \subset Y, K_{n}(X) \subset Y$ for all $K_{n}=K_{\alpha_{n}}, K_{n} y \rightarrow y$ for all $y \in Y$, and $\left\|\left.(S-K+I)\right|_{Y}\right\|>1+\varepsilon / 2$ for all $K \in \operatorname{conv}\left\{K_{1}, K_{2}, \ldots\right\}$. This will contradict the observation above, and complete the proof.

To begin, choose $K_{1}=K_{\alpha_{1}} \in U_{0}$ such that $\left\|K_{1} x-x\right\|<1$ for all $x \in S\left(B_{X}\right)$. Assume that a convex $s_{o p}$ neighbourhood $U_{n-1} \subset U_{n-2}$ (where $U_{-1}=U_{0}$ ) and $K_{n}=K_{\alpha_{n}} \in U_{n-1}$ have been chosen. Consider $S_{\lambda} \in L(X), \lambda=\left(\lambda_{1}, \ldots, \lambda_{n}\right) \in \Lambda_{n}$, defined by $S_{\lambda}=S-\left(\lambda_{1} K_{1}+\cdots+\lambda_{n} K_{n}\right)+I$. For all $\lambda \in \Lambda_{n}$, select $x_{\lambda} \in B_{X}$ such that $\left\|S_{\lambda} x_{\lambda}\right\|>\left\|S_{\lambda}\right\|-\varepsilon / 4$, and denote $C_{n}=\left\{x_{\lambda}: \lambda \in \Lambda_{n}\right\}$. Put

$$
F_{n}=(1+\varepsilon) B_{L(X)}+\operatorname{conv}\left\{K_{1}, \ldots, K_{n}\right\}-S .
$$


Since $F_{n}$ is closed in the $s_{o p}$ and does not contain $I$ (by (1)), there is a convex $s_{o p}$ neighbourhood $U_{n} \subset U_{n-1}$ of $I$ such that $U_{n} \cap F_{n}=\emptyset$, which means

$$
\|S-K+L\|>1+\varepsilon \quad \forall K \in \operatorname{conv}\left\{K_{1}, \ldots, K_{n}\right\}, \quad \forall L \in U_{n} .
$$

Choose $K_{n+1}=K_{\alpha_{n+1}} \in U_{n}$ such that

$$
\left\|K_{n+1} x-x\right\|<\frac{1}{n+1} \quad \forall x \in C_{1} \cup \cdots \cup C_{n} \cup S\left(B_{X}\right) \cup K_{1}\left(B_{X}\right) \cup \cdots \cup K_{n}\left(B_{X}\right) .
$$

Put $Y=\left\{x \in X: \lim K_{n} x=x\right\}$. It is straightforward that $Y$ is closed, $S(X) \subset Y$, and $K_{n}(X) \subset Y, C_{n} \subset Y$ for all $n \in \mathbb{N}$. Moreover, if $K \in \operatorname{conv}\left\{K_{1}, K_{2}, \ldots\right\}$, then $K \in \operatorname{conv}\left\{K_{1}, \ldots, K_{n}\right\}$ for some $n$, and since $\left\|(S-K+I)-S_{\lambda}\right\|<\varepsilon / 4$ for some $\lambda \in \Lambda_{n}$,

$$
\left\|\left.(S-K+I)\right|_{Y}\right\|>\left\|\left.S_{\lambda}\right|_{Y}\right\|-\varepsilon / 4>\left\|S_{\lambda}\right\|-\varepsilon / 2>1+\varepsilon / 2
$$

by (2) and the fact that $I \in U_{n}$.

\section{Kalton-Werner theorem}

Recall (cf. [9]) that a Banach space $X$ is said to have property $(M)$ if

$$
\lim \sup \left\|x+x_{n}\right\|=\lim \sup \left\|y+x_{n}\right\|
$$

whenever $\|x\|=\|y\|$, and $\left(x_{n}\right)$ is a weakly null sequence in $X$; if

$$
\lim \sup \left\|x^{*}+x_{n}^{*}\right\|=\lim \sup \left\|y^{*}+x_{n}^{*}\right\|
$$

whenever $\left\|x^{*}\right\|=\left\|y^{*}\right\|$, and $\left(x_{n}^{*}\right)$ is a weak ${ }^{*}$-null sequence in $X^{*}$, then $X$ is said to have property $\left(M^{*}\right)$. We also need the strong version of property $\left(M^{*}\right)$, which we call property $\left(s M^{*}\right)$, defined by bounded weak ${ }^{*}$-null nets $\left(x_{\lambda}^{*}\right)$ instead of weak ${ }^{*}$-null sequences $\left(x_{n}^{*}\right)$. It is shown in [14], that if $X$ is separable, then properties $\left(M^{*}\right)$ and $\left(s M^{*}\right)$ are equivalent. The Kalton-Werner theorem mentioned above asserts that if a Banach space $X$ is separable, then $K(X)$ is an $M$-ideal in $L(X)$ if and only if $X$ has the MCAP, contains no subspace isomorphic to $\ell_{1}$, and has property $(M)$.

Theorem 3. For a Banach space $X$ the following assertions are equivalent.

(a) $K(X)$ is an $M$-ideal in $L(X)$.

(b) $X$ has the $M C A P$, and has property $\left(s M^{*}\right)$.

(c) $X$ has the $M C A P$, is weakly compactly generated, and has property $\left(M^{*}\right)$.

(d) $X$ has the $M C A P$, contains no subspace isomorphic to $\ell_{1}$, and has property $(M)$.

Proof. (a) $\Longrightarrow$ (b) is well known (cf. [7] and [14] or e.g. [8, p. 299]; cf. [9] for separable case).

(b) $\Longrightarrow\left(\right.$ c). Property $\left(s M^{*}\right)$ implies that $X$ is an $M$-ideal in $X^{* *}$ (cf. [14] or [8, p. 297]; cf. [9] for separable case). But then $X$ is weakly compactly generated (cf. [3] or e.g. [8, p. 142]).

(c) $\Longrightarrow$ (d). Let $Y$ be an arbitrary separable subspace of $X$. We have to show that $Y$ is not isomorphic to $\ell_{1}$, and has property $(M)$. Since $X$ is weakly compactly generated, there exists a separable subspace $Z$ containing $Y$, and a normone projection $P$ of $X$ onto $Z$ (cf. e.g. [2, p. 149]). But then $Z^{*}$ isometrically embeds into $X^{*}$ by means of the formula $z^{*} \in Z^{*} \mapsto z^{*} P \in X^{*}$. This implies that $Z$ also has property $\left(M^{*}\right)$. By [9], since $Z$ is separable, $Z$ has property $(M)$, and is an $M$-ideal in $Z^{* *}$, in particular (cf. [11] or e.g. [8, p. 126]), $Z$ is an Asplund space 
(i.e. every separable subspace of $Z$ has a separable dual). Hence, $Y$ has property $(M)$, and is not isomorphic to $\ell_{1}$.

$(\mathrm{d}) \Longrightarrow(\mathrm{a})$ follows from Theorem 2 and the Kalton-Werner theorem.

Remark. In (c) of Theorem 3, the condition that $X$ is weakly compactly generated clearly may be replaced by the separable complementation property (i.e. every separable subspace of $X$ is contained in a separable subspace which is a range of a norm-one projection on $X$ ).

A Banach space $X$ is said to have the compact approximation property (CAP) if there is a net in $K(X)$ converging strongly to the identity. Since a reflexive space with the CAP has the MCAP (cf. [1] or [5]), we can refine Theorem 3 for reflexive $X$ as follows.

Corollary 4. For a reflexive Banach space $X$, the following assertions are equivalent.

(a) $K(X)$ is an $M$-ideal in $L(X)$.

(b) $X$ has the $C A P$, and has property $\left(s M^{*}\right)$.

(c) $X$ has the $C A P$, and has property $\left(M^{*}\right)$.

(d) $X$ has the $C A P$, and has property $(M)$.

Remark. For separable reflexive spaces $X$, Corollary 4 was obtained in [10]. The equivalence $(\mathrm{a}) \Longleftrightarrow(\mathrm{b})$ of Corollary 4 was established in [12] (using an entirely different proof).

\section{3. $\left(M_{p}\right)$-SPACES}

Let $1 \leq p \leq \infty$. Following [15] (cf. also [8, p. 306]), we say that a Banach space $X$ is an $\left(M_{p}\right)$-space if $K\left(X \oplus_{p} X\right)$ is an $M$-ideal in $L\left(X \oplus_{p} X\right)$. Note that $\left(M_{1}\right)$ spaces are finite dimensional [16], [8, p. 306], and therefore not of interest in the present context. Since every separable subspace of $X \oplus_{p} X$ is contained in $Y \oplus_{p} Y$ for some separable subspace $Y$ of $X$ with the MCAP whenever $X$ has the MCAP (cf. the proof of Theorem 2), the next result follows immediately from Theorem 2.

Corollary 5. Let $1<p \leq \infty$. A Banach space $X$ is an $\left(M_{p}\right)$-space if and only if $X$ has the MCAP, and all separable subspaces of $X$ with the MCAP are $\left(M_{p}\right)$-spaces.

In [10], N. J. Kalton and D. Werner characterized separable $\left(M_{p}\right)$-spaces using the following stronger version of property $(M)$. A Banach space $X$ is said to have property $\left(m_{p}\right)$ if

$$
\lim \sup \left\|x+x_{n}\right\|=\left\|\left(\|x\|, \lim \sup \left\|x_{n}\right\|\right)\right\|_{p}
$$

(where $\|\cdot\|_{p}$ denotes the $\ell_{p}^{2}$-norm) whenever $\left(x_{n}\right)$ is a weakly null sequence in $X$. For separable Banach spaces $X$, the following result was obtained in [10].

Corollary 6. Let $1<p \leq \infty$. For a Banach space $X$, the following assertions are equivalent.

(a) $X$ is an $\left(M_{p}\right)$-space.

(b) $K(X)$ is an $M$-ideal in $L(X)$, and $X$ has property $\left(m_{p}\right)$.

(c) $X$ has the MCAP, contains no subspace isomorphic to $\ell_{1}$, and has property $\left(m_{p}\right)$. 
(d) $X$ has the MCAP, and every separable subspace of $X$ is almost isometric (in the sense of Banach-Mazur distance) to a subspace of an $\ell_{p}$-sum of finitedimensional spaces when $p<\infty$, respectively, to a subspace of $c_{0}$ when $p=\infty$.

Proof. The equivalence (b) $\Longleftrightarrow(\mathrm{c})$ is clear from Theorem 3 since $\left(m_{p}\right)$ implies $(M)$; (c) $\Longleftrightarrow(\mathrm{d})$ is clear from Theorems 3.3 and 3.5 in [10] since considered $\ell_{p^{-}}$ sums are reflexive, and subspaces of $c_{0}$ fail to contain a copy of $\ell_{1}$. The equivalence (a) $\Longleftrightarrow$ (c) is proved in [10] for separable $X$; it extends to the general case by Corollary 5 using the fact that if $X$ has the MCAP, then every separable subspace of $X$ is contained in a separable subspace having the MCAP.

Finally, we come to the most important application of this paper - a characterization of $\left(M_{\infty}\right)$-spaces. The class of $\left(M_{\infty}\right)$-spaces was introduced and studied by $\mathrm{R}$. Payá and W. Werner in [16], where it is proved that $X$ is an $\left(M_{\infty}\right)$-space if and only if $K(Z, X)$ is an $M$-ideal in $L(Z, X)$ for every Banach space $X$. One of the main results of [13] states that a separable Banach space $Y$ with the MCAP is an $\left(M_{\infty}\right)$-space if and only if $K\left(\ell_{1}, Y\right)$ is an $M$-ideal in $L\left(\ell_{1}, Y\right)$. As we now see, this is also true for non-separable spaces.

Corollary 7. A Banach space $X$ is an $\left(M_{\infty}\right)$-space if and only if $X$ has the MCAP, and $K\left(\ell_{1}, X\right)$ is an $M$-ideal in $L\left(\ell_{1}, X\right)$.

Proof. The necessity is clear from the above. The sufficiency immediately follows from Corollary 5 and the result of [13] stated just before Corollary 7 , because the $M$-ideal property of $K\left(\ell_{1}, X\right)$ in $L\left(\ell_{1}, X\right)$ implies that $K\left(\ell_{1}, Y\right)$ is an $M$-ideal in $L\left(\ell_{1}, Y\right)$ for all subspaces $Y$ of $X[13]$.

\section{ACKNOWLEDGMENT}

The author wishes to thank M. Põldvere for interesting discussions on the topic of this paper.

\section{REFERENCES}

1. C.-M. Cho and W. B. Johnson, A characterization of subspaces $X$ of $\ell_{p}$ for which $K(X)$ is an $M$-ideal in $L(X)$, Proc. Amer. Math. Soc. 93 (1985), 466-470. MR 86h:46026

2. J. Diestel, Geometry of Banach Spaces - Selected Topics, Lecture Notes in Math., vol. 485, Springer-Verlag, Berlin, Heidelberg, and New York, 1975. MR 57:1079

3. M. Fabian and G. Godefroy, The dual of every Asplund space admits a projectional resolution of the identity, Studia Math. 91 (1988), 141-151. MR 90b:46032

4. M. Feder and P. Saphar, Spaces of compact operators and their dual spaces, Israel J. Math. 21 (1975), 38-49. MR 51:13762

5. G. Godefroy and P. Saphar, Duality in spaces of operators and smooth norms on Banach spaces, Illinois J. Math. 32 (1988), 672-695. MR 89j:47026

6. R. Haller and E. Oja, Geometric characterizations of positions of Banach spaces in their biduals, Arch. Math. 69 (1997), 227-233. CMP 97:16

7. P. Harmand and A. Lima, Banach spaces which are $M$-ideals in their biduals, Trans. Amer. Math. Soc. 283 (1984), 253-264. MR 86b:46016

8. P. Harmand, D. Werner, and W. Werner, M-ideals in Banach spaces and Banach algebras, Lecture Notes in Math., vol. 1547, Springer-Verlag, Berlin and Heidelberg, 1993. MR 94k:46022

9. N. J. Kalton, M-ideals of compact operators, Illinois J. Math. 37 (1993), 147-169. MR 94b: 46028

10. N. J. Kalton and D. Werner, Property $(M), M$-ideals, and almost isometric structure of Banach spaces, J. reine angew. Math. 461 (1995), 137-178. MR 96m:46022 
11. Å. Lima, On M-ideals and best approximation, Indiana Univ. Math. J. 31 (1982), 27-36. MR 83b: 46021

12. A. Lima, Property $\left(w M^{*}\right)$ and the unconditional metric compact approximation property, Studia Math. 113 (1995), 249-263. MR 96c:46019

13. Å. Lima, E. Oja, T. S. S. R. K. Rao, and D. Werner, Geometry of operator spaces, Michigan Math. J. 41 (1994), 473-490. MR 95h:46027

14. E. Oja, A note on $M$-ideals of compact operators, Acta et Comment. Univ. Tartuensis 960 (1993), 75-92. MR 95a:46026

15. E. Oja and D. Werner, Remarks on $M$-ideals of compact operators on $X \oplus_{p} X$, Math. Nachr. 152 (1991), 101-111. MR 92g:47055

16. R. Payá and W. Werner, An approximation property related to $M$-ideals of compact operators, Proc. Amer. Math. Soc. 111 (1991), 993-1001. MR 91g:46018

17. I. Singer, Bases in Banach spaces II, Editura Acad. R. S. România, Springer-Verlag, Bucureşti, 1981. MR 82k:46024

18. D. Werner, M-ideals and the "basic inequality", J. Approx. Th. 76 (1994), 21-30. MR 95i: 47080

19. W. Werner, Inner M-ideals in Banach algebras, Math. Ann. 291 (1991), 205-223. MR 93b:46094

Institute of Pure Mathematics, Tartu University, Vanemuise 46, EE2400 Tartu, EsTONIA

E-mail address: eveoja@math.ut.ee 